\title{
Observational evidence for stellar-mass black holes
}

\author{
Jorge Casares \\ Instituto de Astrofísica de Canarias, 38200 - La Laguna, Tenerife, Spain \\ email: jcv@iac.es
}

\begin{abstract}
Radial velocity studies of X-ray binaries provide the most solid evidence for the existence of stellar-mass black holes. We currently have 20 confirmed cases, with dynamical masses in excess of $3 M_{\odot}$. Accurate masses have been obtained for a subset of systems which gives us a hint at the mass spectrum of the black hole population. This review summarizes the history of black hole discoveries and presents the latest results in the field.
\end{abstract}

Keywords. Accretion, accretion disks - black hole physics - X-rays: binaries

\section{Introduction}

Theoretical black holes (BHs) have been considered for almost a century, although their first observational evidence was not found until quite recently, during the past 3 decades. Since then BHs have become essential ingredients in the construction of modern astrophysics on all scales, from binaries to galaxies and AGNs. It is stellar-mass BHs that offer us the best opportunity to study these objects in detail. Their implications are wide ranging, from late evolution of massive stars, supernovae models, production of high-energy radiation, relativistic outflows, chemical enrichment in the Galaxy, etc. But still the most compelling evidence for the existence of stellar-mass BHs relies on dynamical arguments, which will be the focus of this review. Section 2 summarizes the first observations of historical BH "candidates", whereas Sect. 3 reviews the most recent discoveries. In Sect. 4 we deal with BH demography and their mass distribution. Finally, we present our conclusions in Section 5 .

\section{Early discoveries: the first BH "candidates"}

In the late 1960s X-ray detectors onboard satellites revolutionized astronomy with the discovery of an unexpected population of luminous X-ray sources in the Galaxy. The energetics (with $L_{\mathrm{x}} \sim L_{\text {Edd }}$ ) together with their short timescale variability (down to milliseconds) lend support to an interacting binary model where X-rays are supplied by accretion onto a collapsed object (Shklovskii 1967). It is now well established that there are two main populations of X-ray binaries: the high mass X-ray binaries (HMXBs), containing O-B supergiant donor stars, and the low mass X-ray binaries (LMXBs), with typically short orbital periods and K-M donors. The optical flux in LMXBs is triggered by reprocessing of the X-rays into the accretion disc whereas in HMXBs it is dominated by the hot supergiant star (see reviews in Charles \& Coe 2006 and McClintock \& Remillard 2006).

Therefore, it was not surprising that one of the first optical counterparts to be identified was the 9th magnitude supergiant star HD 226868, associated with the HMXB Cyg X-1. But, remarkably, it showed radial velocity variations which made it a prime candidate 
for a stellar-mass BH (Webster \& Murdin 1972, Bolton 1972). The supergiant star was shown to move with a velocity amplitude of $\sim 64 \mathrm{~km} \mathrm{~s}^{-1}$ (later refined to $75 \mathrm{~km} \mathrm{~s}^{-1}$ ) in a 5.6 day orbit due to the gravitational influence of an unseen companion (see Fig. 1). The orbital period $P_{\text {orb }}$ and the radial velocity amplitude $K$, combine in the mass function equation $f\left(M_{\mathrm{x}}\right)=K^{3} P_{\mathrm{orb}} / 2 \pi G=M_{\mathrm{x}}^{3} \sin ^{3} i /\left(M_{\mathrm{x}}+M_{\mathrm{c}}\right)^{2}$ which relates the mass of the compact object $M_{\mathrm{x}}$ with that of the companion star $M_{\mathrm{c}}$ and the inclination angle $i$. The mass function $f\left(M_{\mathrm{x}}\right)$ is a lower limit to $M_{\mathrm{x}}$ and, for the case of Cyg X-1 is $0.25 M_{\odot}$. The key factor here is $M_{\mathrm{c}}$ which, for a HMXB, is a large number and has a wide range of uncertainty. If the optical star were a normal O9.7Iab its mass would be $\sim 33 M_{\odot}$ which, for an edge-on orbit $\left(i=90^{\circ}\right)$, would imply a compact object of $\sim 7 M_{\odot}$. However, the optical star is likely to be under-massive for its spectral type as a result of mass transfer and binary evolution, as has been shown to be the case in several neutron star binaries (e.g. Rappaport \& Joss 1983). In fact, it could be under-massive by as much as a factor of 3 given the uncertainty in distance, $\log g$ and $T_{\text {eff }}$. A plausible lower limit of $10 M_{\odot}$, combined with an upper limit to the inclination of $60^{\circ}$, based on the absence of X-ray eclipses, leads to a compact object of $>4 M_{\odot}$ (Bolton 1975).

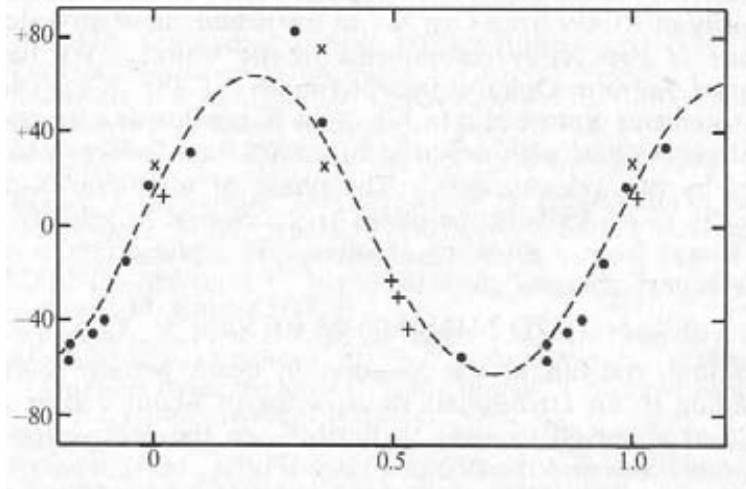

Figure 1. Radial velocity curve of HD 226868, the O9.7Iab companion star in the HMXB Cyg X-1, folded on the 5.6 day orbital period. After Webster \& Murdin (1972).

The importance of this result rests on the fact that there is a maximum mass for neutron stars (NS) to be stable against gravitational collapse (Oppenheimer \& Volkoff 1939). This maximum depends on the equation of state (EoS), which is uncertain in the high density regime because of the poorly constrained many-body interactions. However, Rhoades \& Ruffini (1974) showed that an upper limit of $\sim 3.2 M_{\odot}$ can be derived assuming that causality holds beyond densities where the EoS starts to be uncertain which, at the time, was 1.7 times the density of nuclear matter $\rho_{\mathrm{nm}}=2.7 \times 10^{14} \mathrm{~g} \mathrm{~cm}^{-3}$. More recently, a new limit of $2.9 M_{\odot}$ was obtained using modern EoS which are accurate up to $2 \times \rho_{\text {nm }}$ (Kalogera \& Baym 1996). This can be further boosted by up to $\sim 25 \%$ if the NS rotates close to break-up (Friedman \& Ipser 1987). Therefore, the compact object in Cyg X-1, with $M_{\mathrm{x}} \geqslant 4 M_{\odot}$, is a very strong $\mathrm{BH}$ candidate.

In 1975 the satellite Ariel V detected A0620-00, a new X-ray source which displayed an increase in X-ray flux from non-detection to a record of $\sim 50 \mathrm{Crab}$. It belongs to the class of X-ray transients (XRTs, also called X-ray Novae), a subclass of LMXBs which undergo dramatic episodes of enhanced mass-transfer or "outbursts" triggered by viscous-thermal instabilities in the disc (e.g. King 1999). During outburst, the companion remains undetected because it is totally overwhelmed by the intense optical light from the X-ray heated disc. However, the X-rays switch off after a few months of activity, the reprocessed flux drops several magnitudes into quiescence and the companion star 
becomes the dominant source of optical light. This offers a very special opportunity to perform radial velocity studies of the cool companion and unveil the nature of the compact star. The first detection of the companion in A0620-00 revealed a mid-K star moving in a $7.8 \mathrm{hr}$ period with velocity amplitude of $457 \mathrm{~km} \mathrm{~s}^{-1}$. The implied mass function was $3.2 \pm 0.2 M_{\odot}$, the largest ever measured (McClintock \& Remillard 1986). An absolute $3 \sigma$ lower limit to the mass of the compact star of $3.2 M_{\odot}$ was established by assuming a very conservative low-mass companion of $0.25 M_{\odot}$ and $i<85^{\circ}$, based on the lack of X-ray eclipses. This exceeds the maximum mass allowed for a stable NS and hence it also became a very compelling case for a $\mathrm{BH}$.

\section{From "candidates" to confirmed BHs}

In the 1980s there was a hot debate about the real existence of BHs. On one hand there were 3 strong candidates, the HMXBs Cyg X-1 and LMC X-3 and the transient LMXB A0620-00, all with lower limits to $M_{\mathrm{x}}$ very close to the maximum mass for NS stability. On the other, alternative scenarios were proposed to avoid the need for BHs such as multiple star systems (Bahcall et al. 1974) or non-standard models invoking exotic EoS for condensed matter. An example of the latter are Q-stars, where neutrons and protons are confined by the strong force rather than gravity and can be stable for larger masses (Bahcall et al. 1990). In this context, it was proposed that the "holy grail in the search for black holes is a system with a mass function that is plainly $5 M_{\odot}$ or greater" (McClintock 1986).

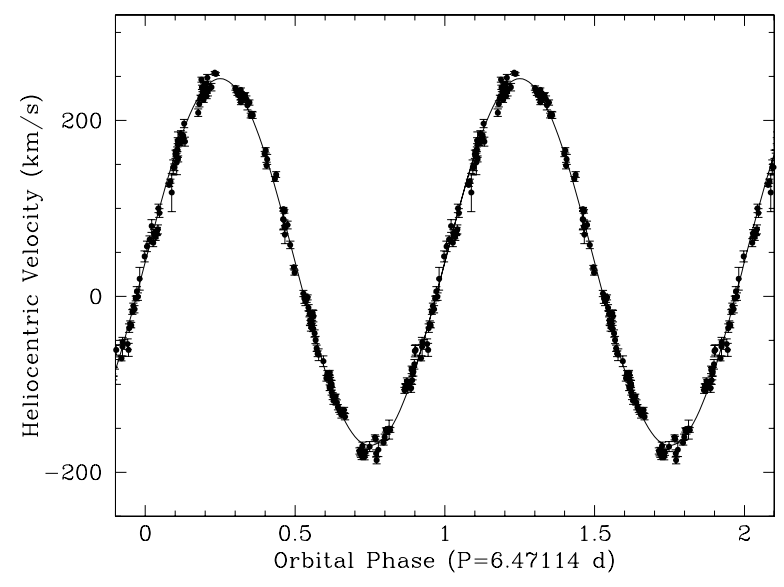

Figure 2. Radial velocity curve of the K0 companion in the transient LMXB V404 Cyg during quiescence. This graph contains velocity points obtained between 1991 and 2005.

In 1989, the X-ray satellite Ginga discovered a new XRT in outburst named GS $2023+338$ (=V404 Cyg). Its X-ray properties drew considerable attention because of the exhibition of a possible luminosity saturation at $\mathrm{L}_{\mathrm{x}} \simeq 10^{39} \mathrm{erg} \mathrm{s}^{-1}$ and dramatic variability (Życki, Done \& Smith 1999). Spectroscopic analysis during quiescence revealed a $\mathrm{K} 0$ star moving with a velocity amplitude of $211 \mathrm{~km} \mathrm{~s}^{-1}$ in a 6.5 day orbit (see Fig. 2). The mass function implied by these numbers is $6.3 \pm 0.3 M_{\odot}$, and hence the compact object must be more massive than $6 M_{\odot}$, independent of any assumption on $M_{\mathrm{c}}$ and $i$ (Casares et al. 1992). This remarkable result established V404 Cyg as the "holy grail" BH for almost a decade. Since then, many other BHs have been unveiled through dynamical studies of XRTs in quiescence, seven others with mass functions also in excess 
Table 1. Confirmed black holes and mass determinations

\begin{tabular}{|c|c|c|c|c|c|}
\hline System & $\begin{array}{c}P_{\text {orb }} \\
{[\text { days }]}\end{array}$ & $\begin{array}{l}f(M) \\
{\left[M_{\odot}\right]}\end{array}$ & $\begin{array}{c}\text { Donor } \\
\text { Spect. Type }\end{array}$ & Classification & $\begin{array}{l}M_{\mathrm{x}}{ }^{\dagger} \\
{\left[M_{\odot}\right]}\end{array}$ \\
\hline GRS 1 & 33.5 & $9.5 \pm 3.0$ & K/M III & LMXB/Trans & $14 \pm 4$ \\
\hline V40 & 1 & 04 & K0 IV & & $12 \pm 2$ \\
\hline Cyg X-1 & 600 & $0.244=$ & 09.7 Iab & HMXB/Persistent & $10 \pm 3$ \\
\hline $\mathrm{LMC}$ & 4.22 & $0.14 \pm$ & 07 III & & $>4$ \\
\hline XTE J & 2.816 & $3.13 \pm$ & B9 III & IMXB/Transient & $7.1 \pm 0.3$ \\
\hline GRO J16 & 2.620 & $2.73 \pm$ & F3/5 IV & & $6.3 \pm 0.3$ \\
\hline BW $\mathrm{Cir}^{b}$ & 2.545 & $5.74 \pm$ & G5 IV & LMXB/Transient & $>7.8$ \\
\hline & 1.75 & $5.8 \pm$ & - & & \\
\hline & 1 & 2.3 & B3 V & HN & $7.6 \pm 1.3$ \\
\hline & 1 & 6.86 & G8/K8 IV & LMY & $9.6 \pm 1.2$ \\
\hline & 125 & 0.25 & A2 V & IMXB $/ \operatorname{Tr}$ & $9.4 \pm 1.0$ \\
\hline $\mathrm{H} 17 \mathrm{C}$ & 0.520 & $4.86 \pm$ & $\mathrm{K} 3 / 7 \mathrm{~V}$ & LMXB/Transient & $6 \pm 2$ \\
\hline GS 11 & 0.433 & $3.01 \pm$ & $\mathrm{K} 3 / 5 \mathrm{~V}$ & 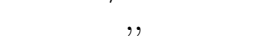 & $7.0 \pm 0.6$ \\
\hline XTE J1859 & 0.382 & & - &, & \\
\hline & 0.345 & $5.01 \pm$ & $\mathrm{K} 3 / 7 \mathrm{~V}$ & & $7.5 \pm 0.3$ \\
\hline & & 2.72 & K4 V & & $11 \pm 2$ \\
\hline & 0.32 & $2.73 \pm$ & K4 V & & \\
\hline & 0.283 & $3.17 \pm$ & K7/M0 V & & $5.2 \pm 0.6$ \\
\hline GRO J042 & 0.212 & $1.19 \pm($ & M2 V & & $4 \pm 1$ \\
\hline XTE J1118+480 & 0.171 & $6.3 \pm 0.2$ & K5/M0 V &, & $6.8 \pm 0.4$ \\
\hline
\end{tabular}

$\dagger$ Masses compiled by Orosz (2003) and Charles \& Coe (2006).

${ }^{a}$ New photometric period of $30.8 \pm 0.2$ days recently reported by Neil, Bailyn \& Cobb (2006). The implied mass function, assuming constant velocity amplitude, would be $8.7 \mathrm{M}_{\odot}$.

${ }^{b}$ Updated after Casares et al. (2007).

${ }^{c}$ Period is uncertain, with another possibility at 0.319 days (see Zurita et al. 2002). This would drop the mass function to $6.18 \mathrm{M}_{\odot}$.

of $5 M_{\odot}$. This has been possible thanks to the improvement in spectrograph performance on a new generation of 10 -m class telescopes over the last decade.

Table 1 presents the current list of 20 confirmed BHs based on dynamical arguments, ordered by orbital period. The case of GRS $1915+105$ is noteworthy, not only because of its long orbital period and large mass function; also because IR spectroscopy was needed to overcome the $>25$ magnitudes of optical extinction and reveal the radial velocity curve of the companion star (Greiner, Cuby \& McCaughrean 2001). It should be noted that, although some systems have mass functions $<3 M_{\odot}$, solid constraints on the inclination and/or $M_{\mathrm{c}}$ can be set which result in $\mathrm{M}_{\mathrm{x}}>3 M_{\odot}$. The great majority of BHs are transients and only 3 show persistent behaviour, the HMXBs Cyg X-1, LMC X-1 and LMC X-3. From the list we also note that a new class of transient X-ray binaries with A-F companions is starting to emerge, the so-called Intermediate Mass X-ray Binaries (IMXBs). It has been proposed that LMXBs may descend from IMXBs through a phase of thermal mass-transfer (Pfahl, Rappaport \& Podsiadlowski 2003), although it is still unclear whether IMXBs and LMXBs represent an evolutionary sequence.

\subsection{A novel technique: fluorescence emission from the irradiated donor}

In addition to the dynamical BHs, there are $\sim 20$ other BH candidates based on their X-ray temporal and spectral behaviour (McClintock \& Remillard 2006). Unfortunately, they have never been seen in quiescence, or they simply become too faint for an optical detection of the companion star. However, a new strategy was devised to allow the extraction of dynamical information in these systems during their X-ray active states. It 
utilises narrow high-excitation emission lines powered by irradiation on the companion star, in particular the strong CIII and fluorescence NIII lines from the Bowen blend at $\lambda \lambda 4630-40$ (see Fig. 3). This technique was first applied to the NS LMXB Sco X-1 and the Doppler shift of the CIII/NIII lines enabled the motion of the donor star to be traced for the first time (Steeghs \& Casares 2002). This was also attempted during the 2002 outburst of the BH candidate GX 339-4, using high-resolution spectroscopy to resolve the sharp NIII/CIII lines. The right panel in Figure 3 shows the radial velocity curve of the NIII lines folded on the 1.76 day orbital period. The orbital solution yields a velocity semi-amplitude of $317 \mathrm{~km} \mathrm{~s}^{-1}$ which defines a strict lower limit to the velocity amplitude of the companion star because these lines arise from the irradiated hemisphere and not the center of mass of the donor. Therefore, a solid lower bound to the mass function is $5.8 M_{\odot}$ which provides compelling evidence for a BH in GX 339-4 (Hynes et al. 2003). And most important, this technique opens an avenue to extract dynamical information from new XRTs in outburst and X-ray persistent LMXBs, which hopefully will help increase the number of $\mathrm{BH}$ discoveries.
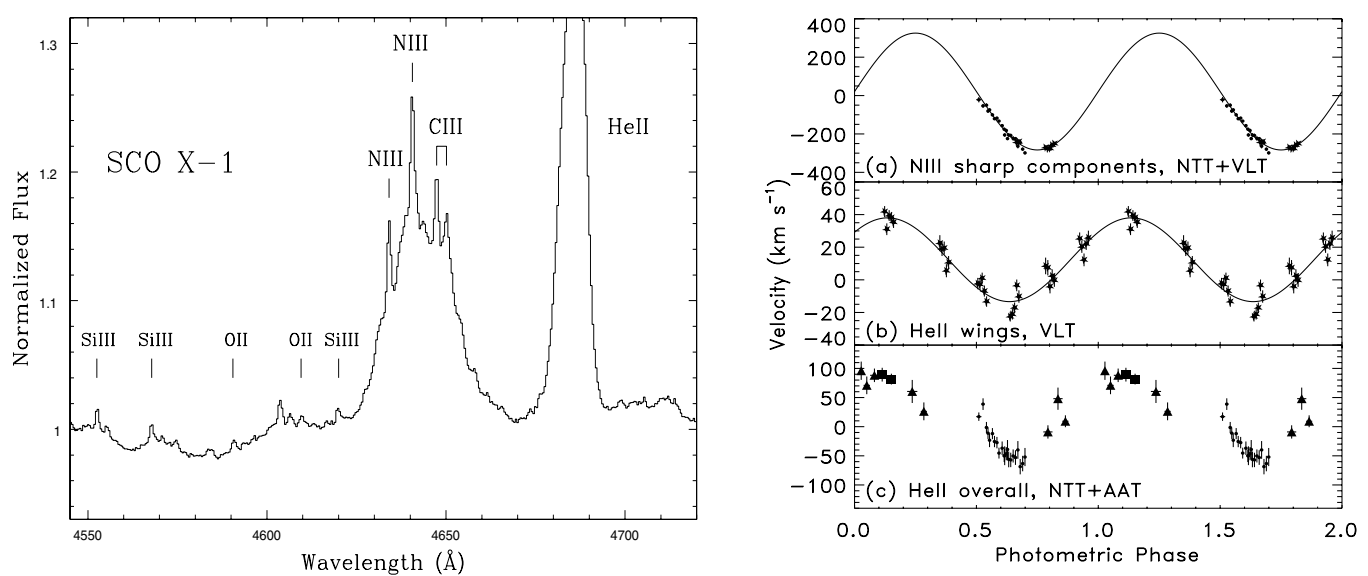

Figure 3. Detecting the companion star in X-ray active LMXBs. Left: the main high excitation emission lines due to by irradiation of the donor star in Sco X-1. Adapted from Steeghs \& Casares (2002). Right: Radial velocities of the NIII lines (top), HeII $\lambda 4686$ (bottom) and the wings of HeII $\lambda 4686$ (middle) in GX 339-4 folded on the 1.76 day orbital period. After Hynes et al. (2003).

\subsection{Further BH signatures}

Aside from dynamical arguments, there are other observations that lend support to the absence of a solid surface and, hence, the BH nature of these accreting compact objects, namely:

- Lack of pulses and Type I X-ray bursts. This has been quantified by Remillard et al. (2006) using observations of dynamical BHs over 9 years of RXTE data. The probability that the non-detection of bursts were consistent with a solid surface is found to be $\sim 2 \times 10^{-7}$.

- The classic colour-colour diagram of X-ray binaries at high accretion rates shows a clear separation in the evolution of NS and BH binaries (Done \& Gierliński 1999). This has been ascribed to the presence of a boundary layer in NS which gives rise to an additional thermal component in the spectrum and drags NS outside the BH region.

- For a given orbital period, quiescent $\mathrm{BH}$ binaries are $\sim 100$ times dimmer than quiescent NS binaries (Menou et al. 1999a). This difference is interpreted as thermal radiation from the NS surface which, for BHs, is advected through the event horizon. 


\section{BH demography}

Despite the handful of confirmed BHs one can try to account for selection effects and estimate the size of the underlying galactic population. To start with, dynamical studies of XRTs indicate that about $75 \%$ contain BHs, i.e. $\mathrm{M}_{\mathrm{x}}>3 M_{\odot}$. Also, the extrapolation of the number of BH XRTs detected since 1975, with outburst duty cycles of $\sim 10-100$ years, suggests that there is a dormant population of $\sim 10^{3} \mathrm{BH}$ binaries. (Romani 1998 and references therein). This is likely to be an underestimate if one accounts for systems with longer recurrence times and a likely population of faint persistent BH LMXBs (Menou et al. 1999b). Even so, incidentally, these numbers are in reasonable agreement with recent population synthesis calculations of BH binaries (Yungelson et al. 2006). On the other hand, stellar evolution models predict a population of about $10^{9}$ stellar-mass BHs in the Galaxy (Brown \& Bethe 1994). Therefore, our observed sample of BH XRTs is just the tip of the iceberg of a large hidden population of which nothing is known. Is it still possible to extract some meaningful information from such a limited sample?
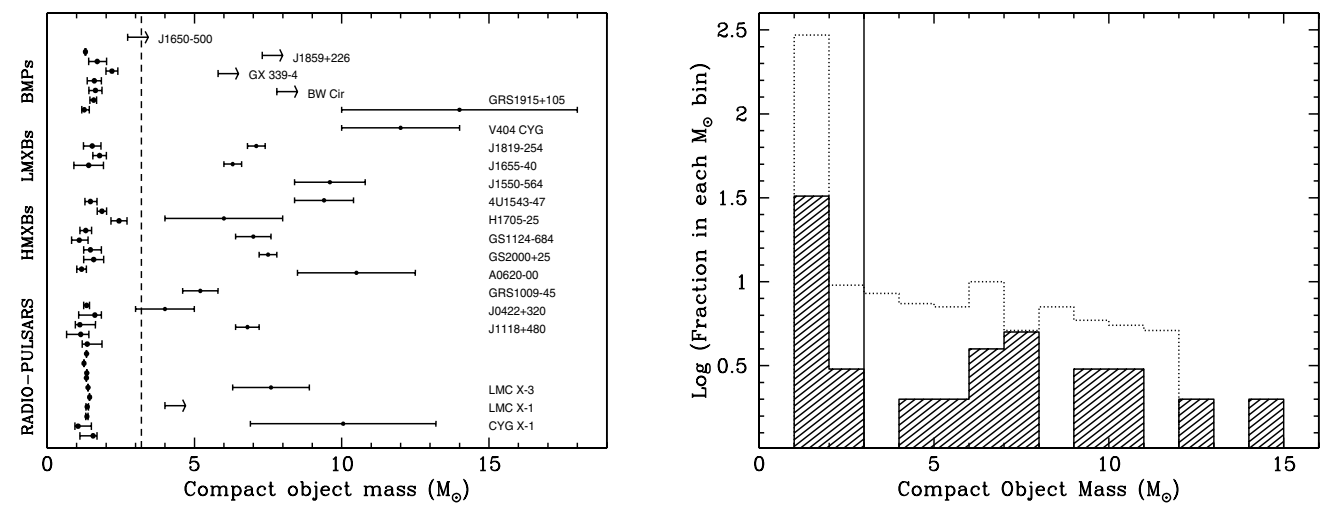

Figure 4. Left: Mass distribution of compact objects in X-ray binaries. Arrows indicate lower limits to $\mathrm{BH}$ masses. Right: observed mass distribution of compact objects in X-ray binaries (shaded histogram), compared to the theoretical distribution computed in Fryer \& Kalogera (2001) for the "Case C + Winds" scenario (dotted line). Mass-loss rates by Woosley, Langer \& Weaver (1995) were used in the computations. The model distribution has been re-scaled for clarity.

The main property of the $\mathrm{BH}$ population is the mass distribution. However, in order to get accurate $\mathrm{BH}$ masses, in addition to the mass function one needs to determine the binary inclination and the mass of the companion star or a related quantity such as the mass ratio. This information can be extracted from two experiments: (i) resolving the rotational broadening $V_{\text {rot }} \sin i$ of the companion's absorption lines, which is correlated with the binary mass ratio and (ii) fitting synthetic models to ellipsoidal lightcurves from which the inclination angle can be determined. This is the classic method to derive masses which has been reviewed in several papers e.g. Casares (2001).

Following this prescription, reliable BH masses have been determined in 15 binaries. These are listed in the last column of Table 1 and displayed in Figure 4, relative to NS masses compiled by Stairs (2004) and Lattimer \& Prakash (2004). The figure nicely shows a mass segregation between the 2 populations of collapsed objects, with NS clustering around $1.4 M_{\odot}$ and $\mathrm{BH}$ masses scattering between 4 and $14 M_{\odot}$. Many more $\mathrm{BH}$ masses and errorbars $\leqslant 10 \%$ are needed before fundamental questions can be addressed such as (i) do BHs masses cluster at a particular value? (ii) what are the edges of the distribution, i.e the minimum and maximum masses for BHs to be formed in X-ray binaries? (iii) is 
there a continuum distribution of masses between NS and BHs? All these questions are intimately related to models of SN and close binary evolution.

Figure 4 also shows the histogram of the current distribution of compact remnant masses, relative to a model distribution computed by Fryer \& Kalogera (2001) which includes mass-loss through winds and binarity effects. The model shows a mass cut at $12 M_{\odot}$ but this seems to be challenged by the compact objects in V404 Cyg and GRS $1915+105$, with $12 \pm 2 M_{\odot}$ and $14 \pm 4 M_{\odot}$ respectively. However, the conflict may be spurious since recent estimates of mass-loss rates in Wolf-Rayet stars suggests that previous determinations were biased too low (Nugis \& Lamers 2000). Also, the model predicts a continuum distribution between NS and BH whereas observations seem to show a paucity of objects at $\sim 3-4 M_{\odot}$. This may be caused by selection effects since low-mass $\mathrm{BHs}$ are expected to be persistent. Recently, compact object masses in the range $2-4 M_{\odot}$ have been reported in 4U1700-37 (Clark et al. 2002), V395 Car (Shahbaz et al. 2004) and LS 5039 (Casares et al. 2005). Although they are assumed to contain NS, none of them has shown X-ray bursts nor pulses. Therefore, they may well be members of the missing low-mass BH population. We are clearly dominated by low number statistics and more observations are required.

\section{Conclusions}

The best observational evidence for the existence of stellar-mass BHs is provided by dynamical studies of X-ray binaries The first solid candidates were the classic X-ray binaries Cyg X-1 and, in particular, A0620-00. BHs became confirmed with the discovery of mass functions in excess of $5-6 M_{\odot}$, the first case being V404 Cyg. Their global X-ray properties (such as the lack of pulsations/bursts, weak quiescent Lx, etc.) also support the presence of an event horizon in these objects.

XRTs are the best hunting ground for new stellar-mass BHs with 17 cases currently known and estimated masses between $4-14 M_{\odot}$. These are only the tip of the iceberg of an estimated dormant population of $\sim 10^{3} \mathrm{BH}$ binaries and $\sim 10^{9}$ stellar-mass BHs in the Galaxy. Clearly many more discoveries and better statistics are essential to derive useful constraints on $\mathrm{BH}$ formation models.

New strategies, aimed at unveiling more quiescent BH transients, and novel techniques, such as the detection of the irradiated donor using high-excitation reprocessed lines, need to be exploited.

\section{Acknowledgements}

I acknowledge useful comments from Phil Charles. I am also grateful for support from the Spanish MCYT grant AYA2002-0036.

\section{References}

Bahcall J. N., Dyson F. J., Katz J. I. \& Pacyńsky B. 1974, ApJ, 189, L17

Bahcall S., Bryan W. \& Selipsky S. B. 1990, ApJ, 362, 251

Bolton C. T. 1972, Nature Phys. Sci., 235, 271

Bolton C. T. 1975, ApJ, 200, 269

Brown G. E. \& Bethe H. A. 1994, ApJ, 423, 659

Casares J. 2001, in: F. C. Lazaro \& M. J. Arevalo (eds.), Binary Stars: Selected Topics on Observations and Physical Processes, LNP 563, p. 277

Casares J., Charles P. A. \& Naylor T. 1992, Nature, 355, 614 
Casares J. et al., 2007, in preparation

Casares J., Ribó M., Ribas I., Paredes J. M., Martí J. \& Herrero A. 2005, MNRAS, 364, 899

Charles P. A. \& Coe M. J. 2006, in: W. H. G. Lewin \& M. van der Klis (eds.), Compact Stellar X-ray Sources, Cambridge Astrophysics Series No. 39 (Cambridge: Cambridge University Press), p. 215

Clark J. S., Goodwin S. P., Crowther P. A., Kaper L. Fairbairn M., Langer N. \& Brocksopp C. 2002, A\&A, 392, 909

Done C. \& Gierliński M. 1999, MNRAS, 342, 1041

Friedman J. L. \& Ipser J.R. 1987, ApJ, 314, 594

Fryer C. L. \& Kalogera V. 2001, ApJ, 554, 548.

Greiner J., Cuby J. G. \& McCaughrean M. J. 2001, Nature, 414, 522

Hynes R. I., Steeghs D., Casares J., Charles P. A. \& O'Brien K. 2003, ApJ, 583, L95

Jonker P. G., Steeghs D., Nelemans G. \& van der Klis M. 2005, MNRAS, 356, 621

Kalogera V. \& Baym G. 1996, ApJ, 470, L61

King A. R. 1999, Phys. Rev., 311, 337

Lattimer J. M. \& Prakash M. 2004, Science, 304, 536

McClintock J. E. 1986, in: K. O. Mason, M. G. Watson \& N. E White (eds.), The Physics of Accretion onto Compact Objects, (Heidelberg: Springer), vol. 266, p. 211

McClintock J. E. \& Remillard R. A. 1986, ApJ, 308, 110

McClintock J. E. \& Remillard R. A. 2006, in: W. H. G. Lewin \& M. van der Klis (eds.), Compact Stellar X-ray Sources (Cambridge University Press: Cambridge), p. 157

Menou K., Esin A. A., Narayan R., Garcia M. R., Lasota J.-P. \& McClintock J. E. 1999a, ApJ, 520,276

Menou K., Narayan R., \& Lasota J.-P. 1999b, ApJ, 513, 811

Neil E. T., Bailyn C. D. \& Cobb B. E. 2006, ApJ, in press (astro-ph/0610480)

Nugis T. \& Lamers H. J. G. L. M. 2000, A\&A, 360, 227

Oppenheimer J. R. \& Volkoff G. M 1939, Phys. Rev., 55, 374

Orosz, J. A. 2003, in: K. A. van der Hucht, A. Herrero \& C. Esteban (eds.) A Massive Star Odyssey, from Main Sequence to Supernova, Proc. IAU Symp. No. 212 (San Francisco: Astronomical Society of the Pacific) p. 365

Pfahl E., Rappaport S. \& Podsiadlowski P. 2003, ApJ, 597, 1036

Rappaport S. A. \& Joss P. C.. 1983, in: W. H. G. Lewin \& E. P. J.. van den Heuve (eds.) Accretion-driven X-ray Sources, (Cambridge: Cambridge University Press), p. 33

Remillard R. A., Dacheng L., Cooper R. L. \& Narayan R. 2006, ApJ, 646, 407

Rhoades C. E. \& Ruffini R. 1974, Phys. Rev. Lett., 32, 324

Romani R. W. 1998, A\&A, 333, 583

Shahbaz T. et al. 2004, ApJ, 616, L123

Shklovskii I. S. 1967, Astron. Zhur., 44, 930

Stairs I. H. 2004, Science, 304, 547

Steeghs D. \& Casares J. 2002, ApJ, 568, 273

Woosley S. E., Langer N. \& Weaver T. A. 1995, ApJ, 448, 315

Webster B. L. \& Murdin P. 1972, Nature, 235, 37

Yungelson L. R. et al. 2006, A\&A, 454, 559

Zurita C. et al. 2002, MNRAS, 334, 999

Życki P. T., Done C. \& Smith D. A. 1999, MNRAS, 309, 561

RAMESH NARAYAN: When you make use of the ellipsoidal model to determine the binary inclination, how do you avoid contamination of the stellar light by the disc light?

Jorge CASAREs: The example I showed is a "textbook" lightcurve of J1655-40 where the hot F6 III companion star totally dominates the optical light. However, for the great majority of black hole binaries (which contain much cooler companions in shorter orbital periods) the contamination by the accretion disc light certainly starts to show up in 
the form of flaring activity and superhumps. It is commonly assumed that the active contribution of the accretion disc light decreases at longer wavelengths and, hence, IR lightcurves are used to estimate the orbital inclination.

Tsevi Mazeh: For HMXB, is it a necessary assumption that the star fills its Roche lobe? If not, the ellipsoidal effect is difficult to model.

Jorge CASAREs: Roche-lobe filling is not a general assumption of the models because HMXBs are normally wind-fed systems. Normally, the ellipsoidal fits are applied with an extra free parameter which is the star filling-factor.

XiaOPeI PAN: Physical parameters of black holes have huge uncertainties. The Space Interferometry Mission (SIM) can provide high-accuracy determination of inclination, mass, distance, etc. The precisions of black-hole masses can be directly determined to $5 \%$. The inclination precision can reach $2 \%$, and the distance of black holes can be measured to better that $2 \%$ by the SIM project. We hope in more collaborations on black hole issues.

Jorge CASARES: The error budget in black-hole mass determination is clearly dominated by uncertainties in the inclination angle and indeed the improvement that the SIM project may provide will be extremely useful.

ANDREW KING: Comment: I would like to reinforce your point about the black holes we detect being the top of an iceberg. Long-period LMXBs have wide separations, big discs, and are all transient. However, the interval between outbursts is so long that we will never detect them at all. We can detect the endpoints of these LMXBs when they contain neutron stars as very wide binaries with millisecond pulsars, but not when they contain black holes. 
